\title{
Effects of Synthetic Jet Array on Turbulent Boundary Layer
}

\author{
Zhixian Ye*, Yiyang Jiang, Yang Zhang, Jianfeng Zou, Yao Zheng \\ School of Aeronautics and Astronautics, Zhejiang University, Hangzhou 310027, China
}

Corresponding Author Email: ye_zhixian@zju.edu.cn

https://doi.org/10.18280/ijht.370327

Received: 1 March 2019

Accepted: 18 June 2019

\section{Keywords:}

synthetic jet array, turbulent boundary

layer, hot-wire, drag reduction

\begin{abstract}
In this paper, streamwise slots are created in the spanwise direction of a test plate with zero pressure gradient, forming a synthetic jet array. Then, a hot-wire constant temperature anemometer (CTA) was adopted to measure the velocities in the turbulent boundary layer at different points in the streamwise and spanwise directions downstream the array. Based on the measured data, the author experimentally explored the effects of the array on the time sequence features and statistical features of the turbulent boundary layer. During the experiment, the array generated unsteady disturbance on the turbulent boundary layer. Then, the spanwise distribution and streamwise attenuation of the disturbance were analyzed through autocorrelation analysis, and the turbulent flow fields before and after the disturbance were compared in terms of mean velocity, fluctuating velocity, frictional resistance, etc. The results show that the synthetic jet array has a drag reduction effect on the turbulent boundary layer within a certain distance in its downstream; the disturbance intensity of the turbulent boundary layer attenuates in the streamwise direction; the synthetic jet will obviously change the flow field structure in the turbulent boundary layer, and raise the bottom of that layer; the excitation effect focuses on the logarithmic region, and extends towards the outer region of the turbulent boundary layer.
\end{abstract}

\section{INTRODUCTION}

Turbulence flow control poses a huge challenge to researchers of fluid mechanics. In turbulence structure, the near-wall streamwise vortex creates streamwise streak in shear transport process. In return, the streamwise streak induces streamwise vortex due to the loss of stability. The two processes form a self-sustaining cycle. The streamwise vortex could cause burst of turbulence and create Reynolds stress [1, $2]$.

In the past few decades, numerous methods have emerged to suppress the near-wall coherent structure and reduce the frictional resistance of the turbulent boundary layer, including riblet wall surface [3-5], large vortex breaker [6-7] and flexible wall [8-9]. Meanwhile, various actuators have been explored to achieve active flow control. In terms of application, these actuators fall into two major categories: separation control and drag reduction. In drag reduction, the steady/unsteady suction/blowing and the synthetic jet can suppress the formation of streamwise vortex, and thus lower the frictional resistance [10-11].

Through direct numerical simulation (DNS), Park and Choi et al. [12] computed the effects of steady blowing through streamwise slot on the flow field, and discovered that blowing could raise the near-wall streamwise vortex, reduce its interaction with streamwise streak, and thus reduce the frictional resistance. The drag reduction effect of unsteady blowing through disturbance of the flow field has also been proved through experiments [13-16]. Kerho et al. [17] adopted unsteady blowing to suppress streamwise streak, and the drag was reduced by $40 \%$ at the most in local areas. Despite the above advantages, there is a major defect with the drag reduction through steady/unsteady suction/blowing: the need of an external gas source.

The synthetic jet requires no additional gas supply structure, for the zero-mass jet can complete blowing with the gas in the flow field. If deployed along the stream, the synthetic jet can produce streamwise vortex under specific excitations, which promotes the interaction between streamwise vortex and streak in the turbulent boundary layer. Lorkowski et al. [18] introduced small-scale disturbances to the flow field with a single synthetic jet actuator, and analyzed how the disturbances affect the turbulent boundary layer. The analysis shows that the disturbances did not change the conduction velocity of the near-wall coherent structure, but raised the structure to a certain extent. These results are a signal of drag reduction. With the aid of the DNS, Lee et al. [19] calculated the effects of a synthetic jet array with four streamwise slotted outlets on the turbulent boundary layer. Despite the failure to achieve drag reduction, their research provide reference for the mechanism of the near-wall coherent structure in the turbulent boundary layer. Rathnasingham et al. and Spalding [20-21] used a three-outlet synthetic jet array to disturb the boundary layer flow, optimized the parameters iteratively, and finally reduced the streamwise fluctuating velocity by $30 \%$, the wall pressure pulsation by $15 \%$ and the net drag by $7 \%$.

The above studies demonstrate the great potential of synthetic jet in drag reduction. In this paper, the drag reduction effects of synthetic jet array are explored in details. The time sequence features and statistical features of the turbulent boundary layer were analyzed under jet disturbance. Besides, the evolution trend of jet disturbance in that layer was summed up. 


\section{METHODOLOGY}

Our experiment was carried out in the low-speed wind tunnel of the School of Aeronautics and Astronautics of Zhejiang University, southeastern China's Zhejiang province. The wind tunnel is a half-closed return tunnel. In the test section $(\mathrm{L} \times \mathrm{W} \times \mathrm{H}: 3.5 \mathrm{~m} \times 1.2 \mathrm{~m} \times 1.2 \mathrm{~m})$, the maximum wind velocity is $70 \mathrm{~m} / \mathrm{s}$, and the turbulence coefficient is $0.04 \sim 0.05 \%$. A StreamLine hot-wire constant temperature anemometer (CTA) (Dantec Dynamics, Denmark) was purchased for our experiment. The CTA was connected to a displacement mechanism atop the test section to accurately control its spatial position. The data in the turbulent boundary layer were measured by a 55P15 boundary layer probe (Dantec Dynamics, Denmark) at the sampling frequency of $64 \mathrm{kHz}$. The sampling lasts $30 \mathrm{~s}$ at each measuring point.
The test plate is made up of four acrylic plates. The test platform is $4 \mathrm{~m}$ in length and $1 \mathrm{~m}$ in width. The acrylic plates were supported by an aluminum alloy frame, ensuring the flatness and levelness of the joints between these plates. To eliminate the wall effect on the flow field, $20 \mathrm{~cm}$-tall vertical acrylic plates were installed on both sides of the platform. On the rear edge of the test platform, an adjustable inclination tail board was installed to keep the pressure gradient of the platform at zero. On the wedge-shaped leading edge, a 40mmwide emery belt and a trip wire were applied for manual turning. The right-handed coordinate system was adopted, with the origin at the intersection of the leading-edge line and the symmetry plane, the $\mathrm{x}$-axis along the stream, the $\mathrm{y}$-axis along the normal direction and the z-axis along the span direction. The test platform is illustrated in Figure 1 below.

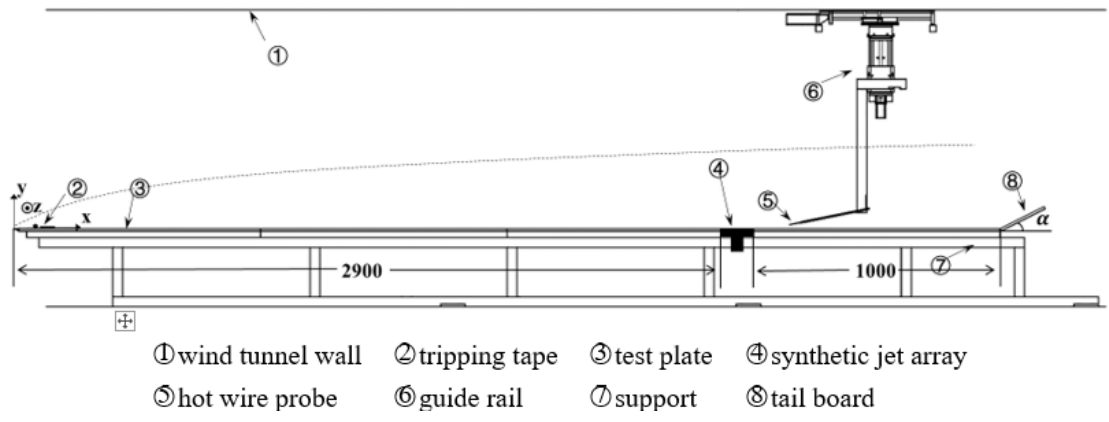

Figure 1. The test platform

The synthetic jet array is shown in Figure 2. The array cover is $500 \mathrm{~mm}$ long and $100 \mathrm{~mm}$ wide. Five actuators were evenly installed beneath the cover, each of which has 2 slotted outlets (length: $30 \mathrm{~mm}$; width: $2 \mathrm{~mm}$ ). The two outlets are $42 \mathrm{~mm}$ apart. The distance from the midpoint between the two outlets (hereinafter referred to as the midpoint) to the leading edge of the platform is $2,950 \mathrm{~mm}$. During the test, the CTA was used to measure 6 streamwise and spanwise points in the downstream of each actuator. The measuring points are $5 \mathrm{~mm}$, $25 \mathrm{~mm}$ or $50 \mathrm{~mm}$ away from the midpoint. This distance is denoted as $\mathrm{x}$. The $\mathrm{x}$ value of $5 \mathrm{~mm}$ was normalized by $\left(x^{+}=\right.$ $x v / u_{\tau}$ ) on the viscous scale $v / u_{\tau}$ without any actuator. The normalized distance $x^{+}$can be expressed as $66,333,666$. For simplicity, the measuring points were numbered as in Figure 3 , where A means measuring points are below the outlet in the downstream, $\mathrm{M}$ means the measuring points are below the midpoint in the downstream, off means the actuators are turned off, and on means the actuators are turned on.
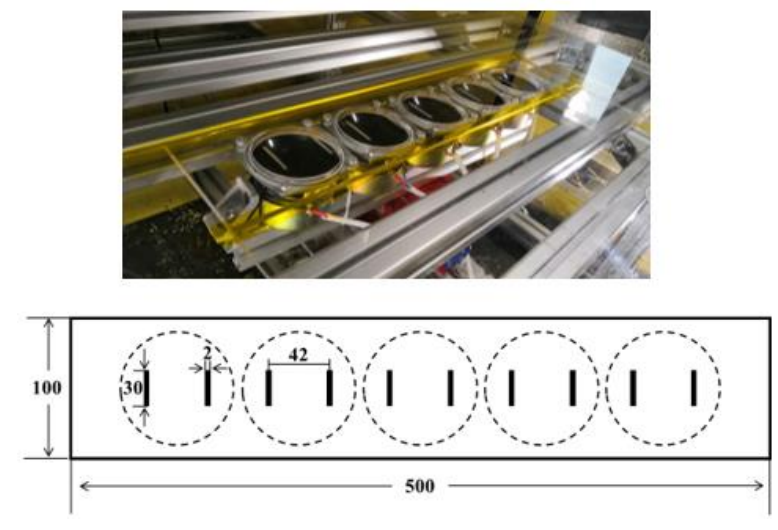

Figure 2. The synthetic jet array and its outlets

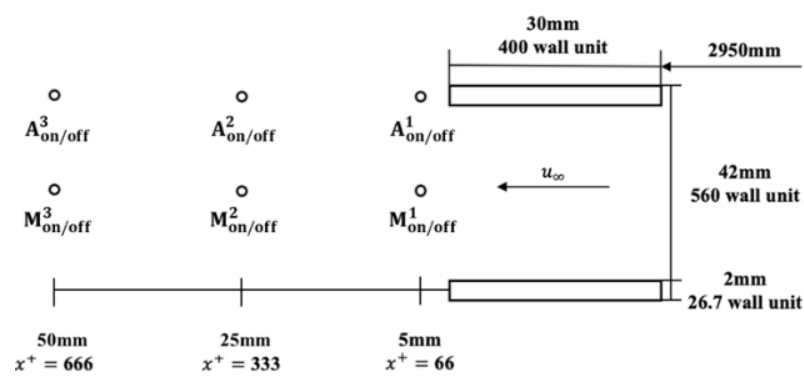

Figure 3. Positions of measuring points

The synthetic jet excitation signal, i.e. the input signal, has a sinusoidal waveform with a peak voltage of $6 \mathrm{~V}$, an excitation frequency of $90 \mathrm{~Hz}\left(f^{+}=f v / u_{\tau}^{2}=0.03\right)$. The outlet velocity was measured at $0.5 \mathrm{~mm}$ above midpoint. The velocity-time curve is shown in Figure 4 . The maximum velocity at the midpoint was $3 \mathrm{~m} / \mathrm{s}$ (corresponding to $A^{+}=\overline{U_{\text {out }}} / u_{\tau}=15$, with $\overline{U_{\text {out }}}$ as the mean outlet velocity). Under these excitation parameters, the actuators can produce a typical synthetic jet.

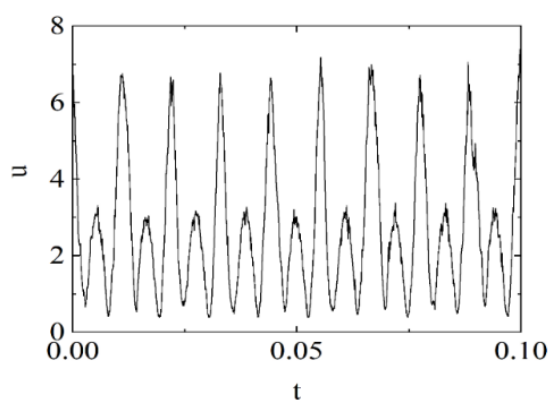

Figure 4. The velocity-time sequence signal measured above the midpoint $(\mathrm{d}=0.5 \mathrm{~mm})$ 


\section{RESULTS ANALYSIS}

\subsection{Fully developed turbulence verification}

The experiment was conducted in a fully developed turbulent boundary layer, under the conditions of zero pressure gradient and smooth surface. To verify whether the turbulence in the test section is fully developed, the velocity distribution in the boundary layer was measured at $3,000 \mathrm{~mm}$ away from the leading edge of the platform without synthetic jet excitation, at the incoming flow velocity $U_{\infty}=6 \mathrm{~m} / \mathrm{s}$ and the turbulent Reynolds number $R e_{\tau} \approx 1,000$. The mean velocity $y^{+}-u^{+}$curve and the fluctuating velocity $y^{+}-u_{r m s}^{+}$curve is displayed in Figure 5.

As shown in Figure 5, the measured data points of the mean velocity curve fell on the test curve of high Reynolds number and the theoretical curve of Spalding formula in existing studies [22]. In the fluctuating velocity curve, $u_{r m s}^{+}$increased with $y^{+}$and peaked at 2.7 when $y^{+} \approx 15$, indicating that the most significant fluctuation appeared at this position. Further increase of $y^{+}$suppressed the $u_{r m s}^{+}$. These trends are consistent with the previous results on turbulent boundary layer. Thus, the turbulence measured in the test section is fully developed.

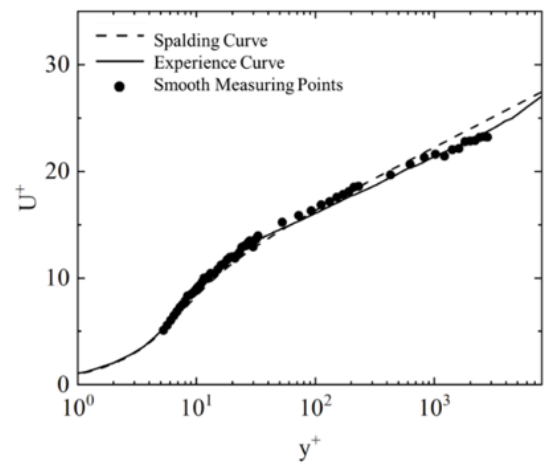

(a) The mean velocity curves

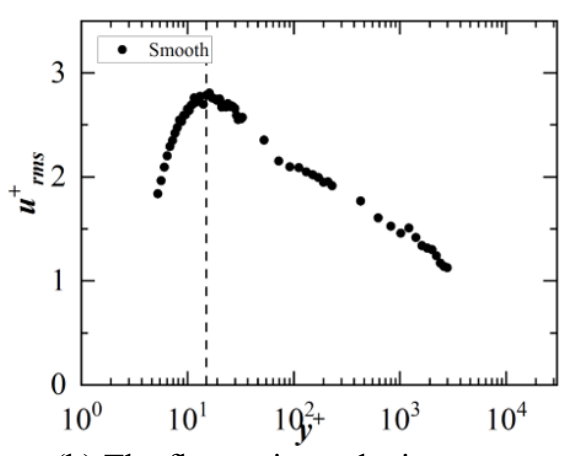

(b) The fluctuating velocity curve

Figure 5. The measured velocities on the boundary layer

\subsection{Effect of synthetic jet on turbulent velocity field}

After the synthetic jet array was installed, the mean velocity of each measuring point was obtained under uncontrolled state and excited state. The mean velocity curves $y^{+}-u^{+}$of the two states are compared in Figure 6, where the solid dots are the results under uncontrolled state, the hollow dots are the results under excited state, and the dotted line is the results of the test platform without the synthetic jet array.
In the downstream under the outlet (A), the linear region still existed and the velocity slightly increased in the logarithmic region, when the synthetic jet array was installed but turned off (uncontrolled state). This is because the flow field is disturbed by slotted outlets. Under the disturbance, the vortex structure moves downward in the logarithmic region of the turbulent boundary layer, and the high velocity region moves downstream. After the synthetic jet array was turned on (excited state), the linear region still conformed to the boundary layer formula, but the velocity significantly declined in the logarithmic region and the outer region. Besides, an obvious inflection point appeared at $y^{+} \approx 300(\mathrm{y} \approx 20 \mathrm{~mm})$, that is, the synthetic jet mainly acted from this region to the outer region of the boundary layer at this moment. The introduction of the synthetic jet helps to increase the thickness of the boundary layer.

In the downstream under the midpoint (M), the velocity was reduced in the logarithmic region after the synthetic jet array was turned on (excited state), but no inflection point appeared as before. This means the actuator has a weak effect on the downstream under the midpoint. In addition, the further away from the midpoint, the velocities decreased more significantly in the logarithmic region and the outer region. Thus, the impacts of the synthetic jet produced by the slotted outlets spread spanwise as it moves further into the downstream.
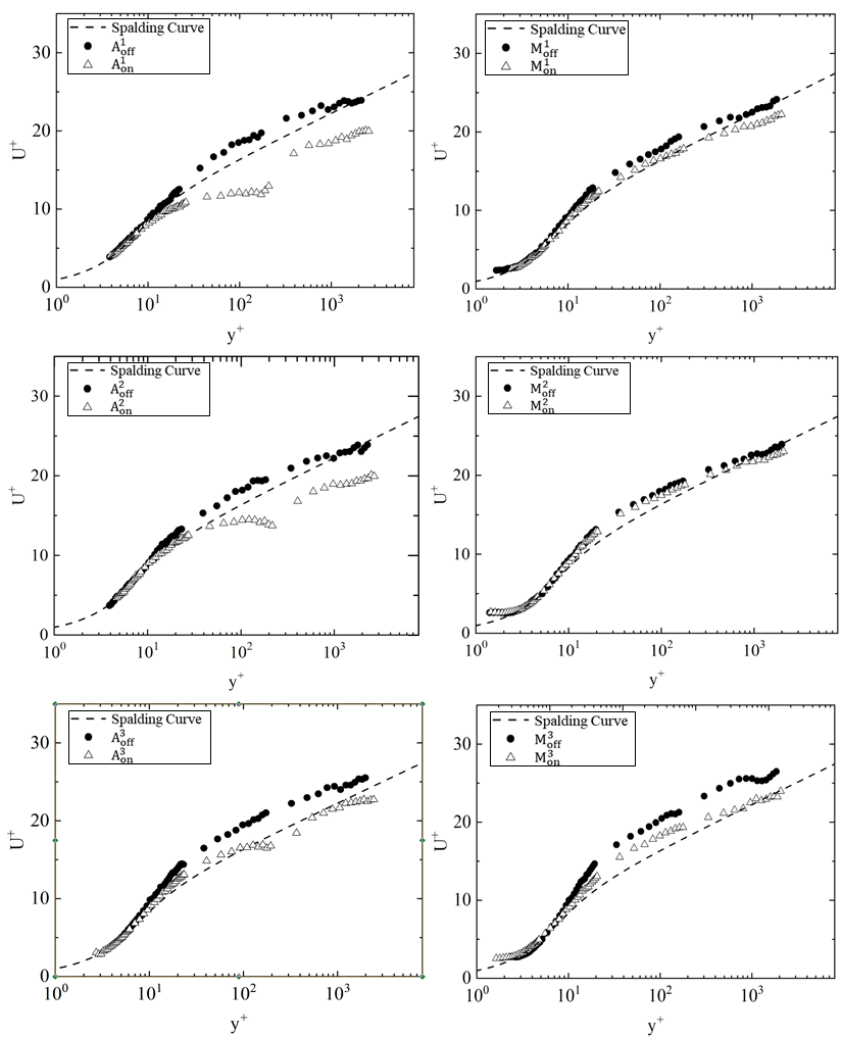

(a) Downstream under the outlet (A); (b) Downstream under the midpoint $(\mathrm{M})$

Figure 6. Mean velocity curves at the measuring points

The fluctuating velocity at each measuring point was measured under uncontrolled state and excited state, with the presence of the synthetic jet array. The fluctuating velocity curves $y^{+}-u_{r m s}^{+}$of the two states are compared in Figure 7, where the vertical dotted line stands for the fluctuating velocity at $y^{+}=15$. 
In the downstream under the outlet (A), the overall fluctuations were greater than those of classic turbulence when the array was turned off (uncontrolled state). In this case, a slight peak appeared $y^{+}=15$ at the point $\mathrm{A}^{1}$, but no peak was observed at $y^{+}=15$ at the other points. In the logarithmic region, the fluctuating velocity did not decline as the classic turbulence, and the high fluctuating velocity extended all the way to the outer region. After the array was turned on (excited state), the fluctuating velocity generally declined, but increased locally at $y^{+} \approx 300$. The high fluctuating velocity also extended to the outer region.

In the downstream under the midpoint $(\mathrm{M})$, the fluctuating velocity still increased and had far-reaching impacts when the array was turned off (uncontrolled state). After the array was turned on (excited state), the fluctuating velocity generally declined, without any local increase at $y^{+} \approx 300$.

After the installation of the array, the fluctuating velocity of the turbulent boundary layer no longer satisfies that of classic turbulence. High fluctuating velocity will appear in large areas and the boundary of turbulence will become more complex.
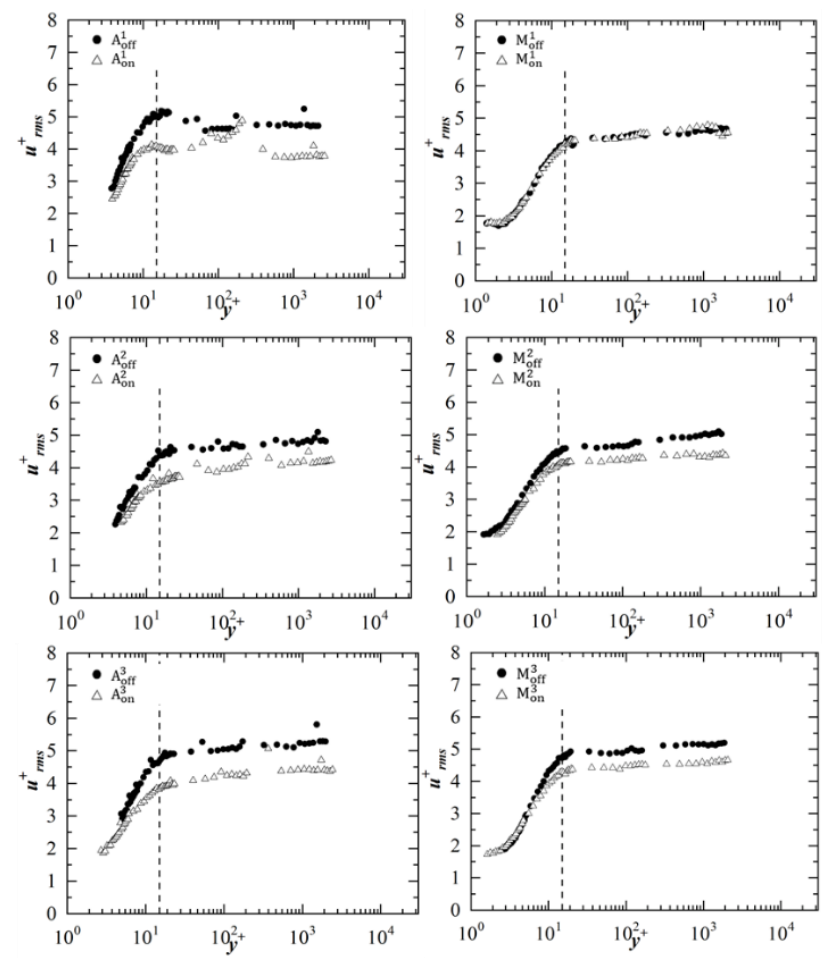

(a) Downstream under the outlet (A); (b) Downstream under the midpoint $(\mathrm{M})$

Figure 7. Fluctuating velocity curves at the measuring points

\subsection{Drag reduction effects of synthetic jet on boundary layer}

The near-wall velocity at each measuring point was measured under uncontrolled state and excited state, after the synthetic jet array had been installed. The near-wall velocity curves $y^{+}-u^{+}$of the two states are compared in Figure 8 .

As shown in Figure 8, the boundary layer velocities obeyed good linear distribution, and could be used to linearly fit the mean velocity in the near-wall region. The oblique lines are the linear trends fitted by the data points, which reflect the features of the linear region. It can be seen that the fitted lines had different slopes after the synthetic jet array was turned on. Thus, the synthetic jet has a certain drag reduction effect.
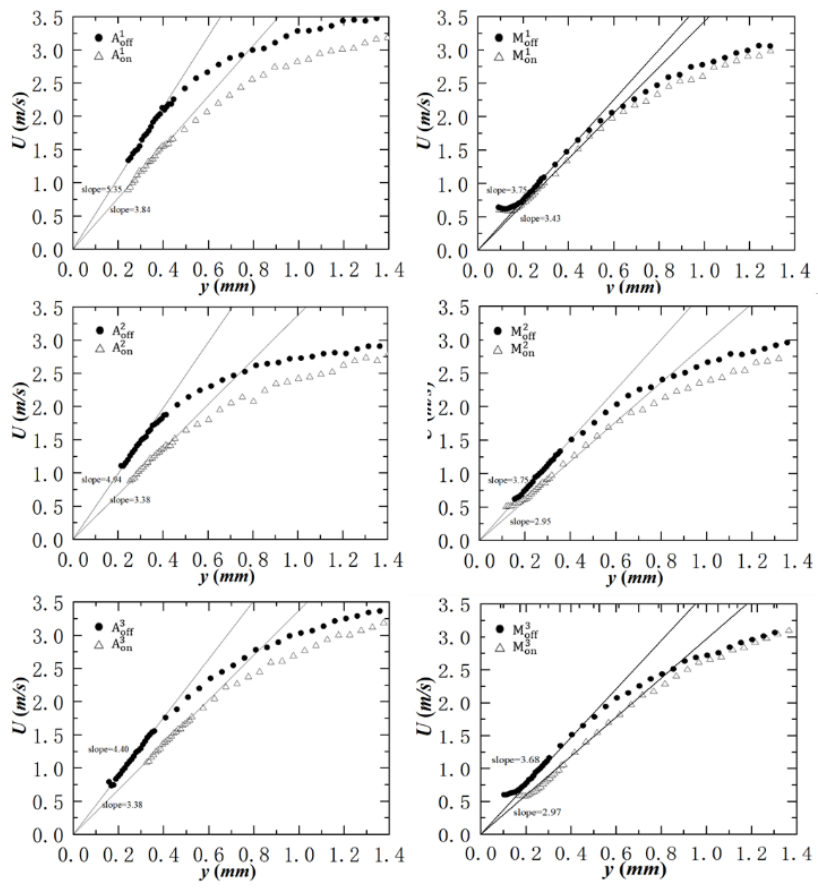

(a) Downstream under the outlet (A); (b) Downstream under the midpoint $(\mathrm{M})$

Figure 8. Comparison of line slopes in the linear region

\subsection{Relative intensity variation of synthetic jet disturbance in turbulent boundary layer}

According to the impacts of the synthetic jet disturbance in turbulent boundary layer, the synthetic jet has a certain drag reduction effect, and the disturbance is a high-frequency periodic disturbance. Figure 9 compares the autocorrelation coefficients of measurement points $\mathrm{A} 1$ and $\mathrm{M} 1$ under the uncontrolled and the excited states, which are the closet to the array. Note that the solid lines are under the uncontrolled state and the dotted lines are under the excited state.
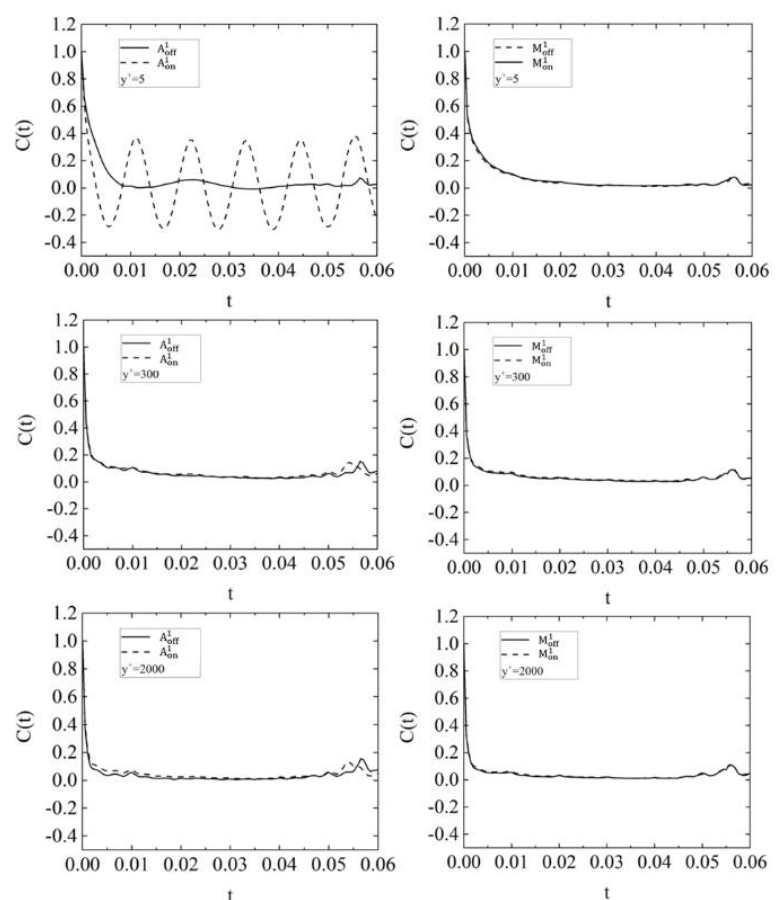

Figure 9. Comparison of autocorrelation coefficients 
As shown in Figure 9, under the excited state, periodic disturbance occurred at A1 with the frequency of $90 \mathrm{~Hz}$. This means the effects of the actuators are obvious at $y^{+}=5$. However, the autocorrelation coefficients showed insignificant changes at $y^{+}=300$ and $y^{+}=2,000$ after the array was turned on.

At the point M1, the activation of the array did not cause obvious changes to the autocorrelation coefficients at $y^{+}=$ $5, y^{+}=300$ or $y^{+}=2,000$. Thus, the effects of the array cannot reach this measuring point.

Figure 10 compares the power spectral curves of A1 and M1 under the uncontrolled and excited state. Note that the solid curves are the results under the controlled state and the dotted curves are the results under the excited curves.

As shown in Figure 10, under the excited state, an obvious peak appeared at $\mathrm{f}=90 \mathrm{~Hz}$, that is, effects of the actuators are obvious at $y^{+}=5$. Meanwhile, the power specturm curves raised in general, revealling the strong effects of the array. However, the power specturm curves were not changed significantly at $y^{+}=300$ or $y^{+}=2,000$ after the array was turned on, neither did any peak appear at $\mathrm{f}=90 \mathrm{~Hz}$.

At the point M1, the activation of the array did not cause obvious changes or peaks to the power spectrum curves at $y^{+}=5, y^{+}=300$ or $y^{+}=2,000$. The variation in the power spectrum shows that the array mainly affects the logarithmic region.
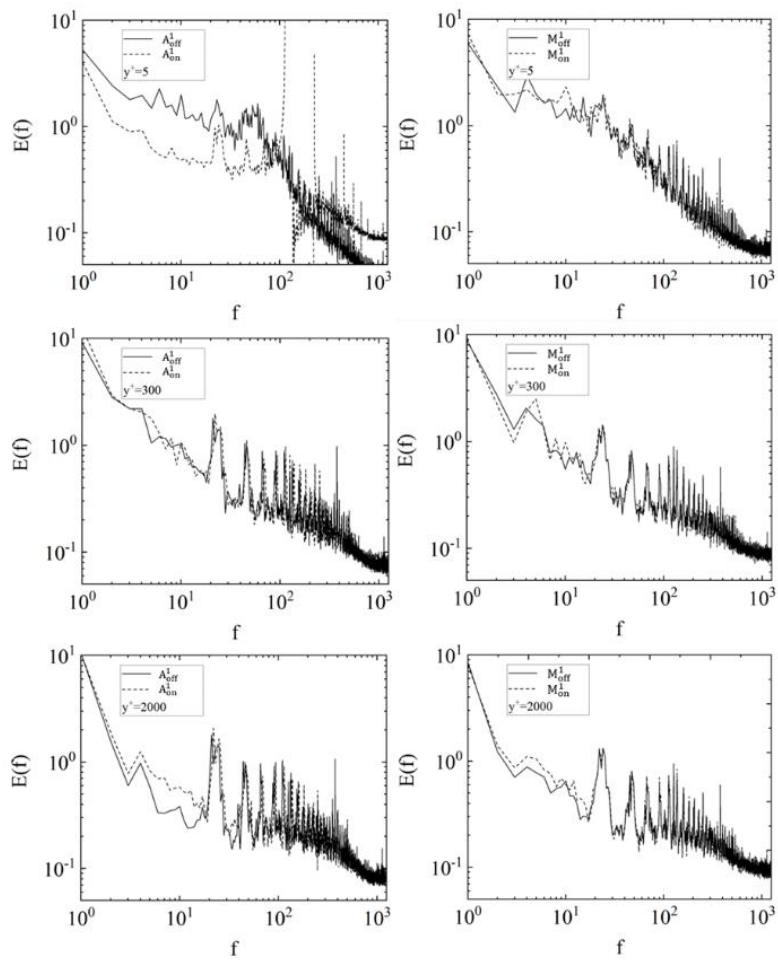

Figure 10. Comparison of power spectrum curves

\section{CONCLUSIONS}

This paper uses a synthetic jet array to disturb the fully developed turbulent boundary layer, and measures the boundary layer velocities with a hot-wire CTA. Based on the measured results, the author explored the effects of the array on the time sequence features and statistical features in the near-wall region of the turbulent boundary layer. The main conclusions are as follows:
(1) The test turbulence was proved to be fully developed. For the turbulent boundary layer, the fitted results in the linear region were contrasted with the empirical formula of the logarithmic region, revealing the accurate velocity distribution relative to the wall position. This provides supports to the accurate measurement of velocity signals.

(2) The setting of slotted outlets in the array can change some features of the turbulent flow field. specifically, the mean velocity and fluctuating velocity will increase in the logarithmic region, that is, the slotted outlets can raise the boundary layer; after the array is turned on, the mean velocity and fluctuating velocity will both decline in the turbulent boundary layer, which thickens the viscous sublayer and reduces the frictional resistance of the wall.

(3) The analysis on autocorrelation coefficients and power spectrum curves reflects the evolution of the synthetic jet disturbance in the streamwise and spanwise directions. The disturbance is stronger under the outlet in the downstream than under the midpoint in the downstream or in the spanwise direction, because the disturbance attenuates faster in the latter positions.

(4) The disturbance of the array does not affect the outer region of the turbulent boundary layer. Considering frictional resistance, it can be seen that the slotted outlets of the actuators change the flow field structure of the boundary layer. Once the array is turned on, the near-wall structure will be raised higher than the other regions, and the coherent structure in the logarithmic region will be severely affected.

\section{ACKNOWLEDGMENT}

The authors would like to acknowledge the financial support received from the project "Drag Reduction via Turbulent Boundary Layer Flow Control (DRAGY)". The DRAGY project (April 2016 - March 2019) is a China-EU Aeronautical Cooperation project, which is co-funded by Ministry of Industry and Information Technology (MIIT), China, and Directorate-General for Research and Innovation (DG RTD), European Commission.

\section{REFERENCES}

[1] Gad-el-Hak, M. (2007). Flow control: passive, active, and reactive flow management. Cambridge University Press. https://doi.org/10.1017/CBO9780511529535

[2] Choi, K.S. (2001). Turbulent drag-reduction mechanisms strategies for turbulence management. In Turbulence Structure and Modulation, 415: 161-212. https://doi.org/10.1007/978-3-7091-2574-8_6

[3] Walsh, M.J., Lindemann, A.M. (1984). Optimization and application of riblets for turbulent drag reduction. In 22nd Aerospace Sciences Meeting, pp. 347-347. https://doi.org/10.2514/6.1984-347

[4] Choi, K.S. (1989). Near-wall structure of a turbulent boundary layer with riblets. Journal of Fluid Mechanics, 208: 417-458. https://doi.org/10.1017/S0022112089002892

[5] Segawa, T., Mizunuma, H., Murakami, K., Li, F.C., Yoshida, H. (2007). Turbulent drag reduction by means of alternating suction and blowing jets. Fluid Dynamics Research, 39(7): https://doi.org/10.1016/j.fluiddyn.2007.01.002 
[6] Bertelrud, A., Truong, T., Avellan, F. (1982). Drag reduction in turbulent boundary layers using ribbons. In 9th Atmospheric Flight Mechanics Conference, pp. 1370-1370. https://doi.org/10.2514/6.1982-1370

[7] Bandyopadhyay, P.R. (1986). Mean flow in turbulent boundary layers disturbed to alter skin friction. Journal of Fluids Engineering, 108(2): 127-140. https://doi.org/10.1115/1.3242552

[8] Guezennec, Y.G., Nagib, H.M. (1990). Mechanisms leading to net drag reduction in manipulated turbulent boundary layers. AIAA Journal, 28(2): 245-252. https://doi.org/10.2514/3.10381

[9] Gad-el-Hak, M. (2002). Compliant coatings for drag reduction. Progress in Aerospace Sciences, 38(1): 77-99. https://doi.org/10.1016/S0376-0421(01)00020-3

[10] Gad-el-Hak, M., Blackwelder, R.F. (1989). Selective suction for controlling bursting events in a boundary layer. AIAA Journal, 27(3): 308-314. https://doi.org/10.2514/3.10113

[11] Myose, R.Y., Blackwelder, R.F. (1995). Control of streamwise vortices using selective suction. AIAA Journal, 33(6): 1076-1080. https://doi.org/10.2514/3.12667

[12] Park, J., Choi, H. (1999). Effects of uniform blowing or suction from a spanwise slot on a turbulent boundary layer flow. Physics of Fluids, 11(10): 3095-3105. https://doi.org/10.1063/1.870167

[13] Park, S.H., Lee, I., Sung, H.J. (2001). Effect of local forcing on a turbulent boundary layer. Experiments in Fluids, 31(4): 384-393. https://doi.org/10.1007/s003480100305

[14] Park, Y.S., Park, S.H., Sung, H.J. (2003). Measurement of local forcing on a turbulent boundary layer using PIV. Experiments in Fluids, 34(6): 697-707. https://doi.org/10.1007/s00348-003-0604-2

[15] Tardu, S.F. (2001). Active control of near-wall turbulence by local oscillating blowing. Journal of Fluid Mechanics, 439: 217-253. https://doi.org/10.1017/S0022112001004542

[16] Gad-el-Hak, M., Blackwelder, R.F. (1989). Selective suction for controlling bursting events in a boundary layer. AIAA Journal, 27(3): 308-314. https://doi.org/10.2514/3.10113

[17] Kerho, M. (2002). Active reduction of skin friction drag using low-speed streak control. In 40th AIAA Aerospace Sciences Meeting and Exhibit, pp. 271-271. https://doi.org/10.2514/6.2002-271

[18] Lorkowski, T., Rathnasingham, R., Breuer, K., Lorkowski, T., Rathnasingham, R., Breuer, K. (1997). Small-scale forcing of a turbulent boundary layer. In 4th Shear Flow Control Conference, pp. 1792-1792. https://doi.org/10.2514/6.1997-1792

[19] Lee, C., Goldstein, D. (2001). DNS of micro jets for turbulent boundary layer control. In 39th Aerospace Sciences Meeting and Exhibit, pp. 1013-1013. https://doi.org/10.2514/6.2001-1013

[20] Rathnasingham, R., Breuer, K.S. (1997). System identification and control of a turbulent boundary layer. Physics of Fluids, 9(7): 1867-1869. https://doi.org/10.1063/1.869337

[21] Rathnasingham, R., Breuer, K.S. (2003). Active control of turbulent boundary layers. Journal of Fluid Mechanics, 495: 209-233. https://doi.org/10.1017/S0022112003006177

[22] Spalding, D.B. (1961). A single formula for the "law of the wall”. Journal of Applied Mechanics, 28(3): 455-458. https://doi.org/10.1115/1.3641728 